\title{
SNP-Based Differentiation of Phytophthora infestans Clonal Lineages Using Locked Nucleic Acid Probes and High-Resolution Melt Analysis
}

\begin{abstract}
Zachariah R. Hansen, Plant Pathology and Plant-Microbe Biology Section, School of Integrative Plant Science, Cornell University, NYSAES, Geneva, NY, 14456; Brian J. Knaus, Horticultural Crops Research Laboratory, United States Department of Agriculture-Agricultural Research Service, (USDA-ARS) Corvallis, OR, 97331; Javier F. Tabima, Botany and Plant Pathology, Oregon State University, Corvallis, 97331; Caroline M. Press, Horticultural Crops Research Laboratory, USDA-ARS; Howard S. Judelson, Department of Plant Pathology and Microbiology, University of California, Riverside, 92521; Niklaus J. Grünwald, Plant Pathology and Plant-Microbe Biology Section, School of Integrative Plant Science, Cornell University; Horticultural Crops Research Laboratory, USDA-ARS; and Botany and Plant Pathology, Oregon State University; and Christine D. Smart, Plant Pathology and Plant-Microbe Biology Section, School of Integrative Plant Science, Cornell University
\end{abstract}

\begin{abstract}
Hansen, Z. R., Knaus, B. J., Tabima, J. F., Press, C. M., Judelson, H. S., Grünwald, N. J., and Smart, C. D. 2016. SNP-based differentiation of Phytophthora infestans clonal lineages using locked nucleic acid probes and high-resolution melt analysis. Plant Dis. 100:1297-1306.

Phytophthora infestans, the cause of the devastating late blight disease of potato and tomato, exhibits a clonal reproductive lifestyle in North America. Phenotypes such as fungicide sensitivity and host preference are conserved among individuals within clonal lineages, while substantial phenotypic differences can exist between lineages. Whole $P$. infestans genomes were aligned and single nucleotide polymorphisms (SNPs) identified as targets for the development of clonal-lineage-specific molecular diagnostic tools. Informative SNPs were used to develop highresolution melt (HRM) assays and locked nucleic acid (LNA) probes to differentiate lineage US-23, the predominant lineage in the Eastern United States for the past several years, from three other U.S. lineages.

Three different primer pairs targeting one to three SNPs were capable of separating lineage US-23 from lineages US-8, US-11, and US-24 using HRM analysis. A fourth HRM primer pair targeted a highly variable genomic region containing nine polymorphisms within $63 \mathrm{bp}$. These primers separated US-23, US-11, and US-8 plus US-24 into three separate groups following HRM analysis but did not separate US-8 from US-24. Additionally, two LNA probes were designed to target a portion of the $P$. infestans genome containing two SNPs diagnostic for US-23. A single multiplex quantitative polymerase chain reaction assay containing both differentially labeled LNA probes differentiated individuals belonging to lineage US-23 from those belonging to US-8, US-11, and US-24.
\end{abstract}

Late blight, caused by Phytophthora infestans, has been an economically important disease of potato since at least the midnineteenth century, when it destroyed Irish potato crops, and continues to be a major constraint on potato and tomato production (Fry et al. 2015; Haverkort et al. 2008; Turner 2005). Our ability to effectively manage the disease is dependent on our knowledge of certain fundamental pathogen characteristics, such as host preference and fungicide sensitivity. $P$. infestans is a heterothallic oomycete; thus, individuals belonging to two different mating types (A1 and A2) must come in contact for sexual reproduction to occur (Flier et al. 2001). In the United States, $P$. infestans has remained asexual since it was first reported in the nineteenth century, with the exception of two ephemerally sexual populations in the northwest in 1993 (Gavino et al. 2000) and the northeast in 2010 and 2011 (Danies et al. 2014). Populations of $P$. infestans observed in a given year in the United States are typically made up of one or more clonal lineages, which comprise clonal descendants of one common ancestor. Occasionally, novel lineages are thought to migrate from asexual or sexual populations elsewhere such as Mexico that can admix with or displace currently existing lineages (Goodwin et al. 1994a; Goss et al. 2014). Following the introduction of a novel genotype, clonal lineages proliferate through asexual reproduction and are dispersed by wind-blown sporangia or movement of infected plant tissue. Some lineages such as US-1 persist for decades or even centuries (Goodwin et al. 1994b). Others, such as US-22, are relatively short-lived and are observed for a few years or less (Fry et al. 2013).

It is well-documented that $P$. infestans individuals belonging to a clonal lineage tend to share phenotypic characteristics, such as

Accepted for publication 3 December 2015.

http://dx.doi.org/10.1094/PDIS-11-15-1247-RE

(C) 2016 The American Phytopathological Society fungicide sensitivity and host preference, while individuals from different lineages can vary greatly in such characteristics (Danies et al. 2013; Hu et al. 2012; Legard et al. 1995). For example, lineages US-8 and US-11 were found to have resistance to the widely used phenylamide fungicide mefenoxam, whereas lineages US-23 and US-24 are currently sensitive (Danies et al. 2013; Goodwin et al. 1998). Lineages also vary with regard to host preference; US-11 and US-23 are both aggressive pathogens of potato and tomato, whereas US-8 and US-24 are much more aggressive on potato than tomato (Danies et al. 2013; Fry and Goodwin 1997; Fry et al. 2013). For this reason, knowledge about the identity of a given isolate's lineage has direct disease management implications.

Decreasing sequencing costs now make it feasible to sequence and align multiple genomes to identify informative single nucleotide polymorphisms (SNPs), which can be used as the basis for molecular diagnostic assays. SNP-based assays offer an additional tool to add to other molecular diagnostic assays, such as simple sequence repeat (SSR) analysis, which is currently used for $P$. infestans lineage genotyping (Hu et al. 2012; Lees et al. 2006). SSR analysis has the distinct advantage over SNP-based diagnostic assays of being able to identify novel alleles, whereas SNP-based diagnostic assays typically only apply to specific, known alleles. However, SSR analysis can be less accessible and, therefore, less feasible for broad scale adoption by diagnostic laboratories.

High-resolution melt (HRM) analysis is a technique which is capable of differentiating genotypes based on the presence of SNPs. HRM takes advantage of differential melting temperatures (Tm) between the bonds formed by nucleotides in double-stranded DNA. Following amplification of target double-stranded DNA using quantitative polymerase chain reaction (qPCR), double-stranded DNA is melted into single-stranded DNA by increasing the temperature in $0.2^{\circ} \mathrm{C}$ increments. These small increments allow for the detection of small differences in Tm which correspond to polymorphisms in the amplified target DNA (Reed et al. 2007). HRM analysis has been 
used to discriminate between fungicide-sensitive and -resistant isolates of the white mold pathogen Sclerotinia sclerotiorum (Lehner et al. 2015), as well as the closely related hop and cucurbit downy mildew pathogens Pseudoperonospora humuli and P. cubensis (Summers et al. 2015).

Locked nucleic acids (LNAs) are another technology capable of differentiating genotypes based on SNPs. LNAs have unique chemical properties that, when incorporated into DNA probes, increase the stability of the resulting DNA duplex when bound to the complementary target sequence. This makes LNA probes highly sensitive to mismatch pairing and, therefore, capable of specific genotypic discrimination based on as few as one SNP (Braasch and Corey 2001; Johnson et al. 2004; Koshkin et al. 1998). For identification of multiple genotypes in a single multiplex reaction, allele-specific probes can be labeled with different reporter dyes, allowing for the rapid identification of genotypes following qPCR. LNA probes have been used in diagnostics for Meloidogyne enterolobii, a quarantined root-knot nematode (Kiewnick et al. 2015). They have also been used for differentiating the aforementioned downy mildew pathogens $P$. humuli and $P$. cubensis (Summers et al. 2015).

The goal of this study was to develop methods for differentiating the currently predominant clonal lineage of Phytophthora infestans

Table 1. Phytophthora infestans isolates used in high-resolution melt and locked nucleic acid tests

\begin{tabular}{|c|c|c|c|c|}
\hline $\begin{array}{l}\text { Isolate } \\
\text { code }\end{array}$ & $\begin{array}{l}\text { Clonal } \\
\text { lineage }\end{array}$ & $\begin{array}{c}\text { Collection } \\
\text { year }\end{array}$ & Collection source $^{a}$ & Host \\
\hline $0882^{b}$ & US-8 & 2008 & Unknown & Unknown \\
\hline $0982^{\mathrm{b}}$ & US-8 & 2009 & Wyoming Co, NY & Potato \\
\hline 1083 & US-8 & 2010 & Shelburne, ON, Canada & Potato \\
\hline 1084 & US-8 & 2010 & Shelburne, ON, Canada & Potato \\
\hline 1087 & US-8 & 2010 & Wayne Co, NY & Potato \\
\hline 1181 & US-8 & 2011 & Erie Co, PA & Potato \\
\hline 1183 & US-8 & 2011 & Pasco, WA & Potato \\
\hline $1185^{\mathrm{b}}$ & US-8 & 2011 & Othello, WA & Potato \\
\hline $1281^{\mathrm{b}}$ & US-8 & 2012 & Painter, VA & Potato \\
\hline $1381^{\mathrm{b}}$ & US-8 & 2013 & Erie Co, PA & Potato \\
\hline $11111^{\mathrm{b}}$ & US-11 & 2011 & Oneida Co, NY & Tomato \\
\hline 11112 & US-11 & 2011 & San Joaqin Co, CA & Tomato \\
\hline $11114^{\mathrm{b}}$ & US-11 & 2011 & Corvallis, OR & Tomato \\
\hline $11115^{\mathrm{b}}$ & US-11 & 2011 & San Joaqin Co, CA & Tomato \\
\hline 11116 & US-11 & 2011 & Linn Co, OR & Tomato \\
\hline 11117 & US-11 & 2011 & Linn Co, OR & Tomato \\
\hline 11118 & US-11 & 2011 & Linn Co, OR & Tomato \\
\hline $12112^{\mathrm{b}}$ & US-11 & 2012 & Collier Co, FL & Tomato \\
\hline $12114^{b}$ & US-11 & 2012 & Skagit Co, WA & Tomato \\
\hline 12116 & US-11 & 2012 & Skagit Co, WA & Tomato \\
\hline $12232^{\mathrm{b}}$ & US-23 & 2012 & Jupiter, FL & Tomato \\
\hline 1237 & US-23 & 2012 & Waukesha Co, WI & Tomato \\
\hline $112317^{\mathrm{b}}$ & US-23 & 2011 & Berks Co, PA & Tomato \\
\hline 112322 & US-23 & 2011 & Bedford, PA & Tomato \\
\hline $122312^{b}$ & US-23 & 2012 & Suffolk Co, NY & Tomato \\
\hline $122322^{b}$ & US-23 & 2012 & Columbia Co, NY & Tomato \\
\hline 122329 & US-23 & 2012 & Morris Co, NJ & Tomato \\
\hline 12237 & US-23 & 2012 & Belvidere, NC & Tomato \\
\hline 132317 & US-23 & 2013 & Lancaster Co, PA & Tomato \\
\hline $132348^{b}$ & US-23 & 2013 & Miami Co, IN & Tomato \\
\hline $11241^{\mathrm{b}}$ & US-24 & 2011 & North Dakota & Potato \\
\hline $1249^{\mathrm{b}}$ & US-24 & 2011 & North Dakota & Potato \\
\hline $1273^{\mathrm{b}}$ & US-24 & 2011 & Freeville, NY & Potato \\
\hline $1250^{\mathrm{b}}$ & US-24 & 2011 & Corvallis, OR & Tomato \\
\hline 1339 & US-24 & 2011 & George Co, WA & Potato \\
\hline 1345 & US-24 & 2010 & Montana & Potato \\
\hline $1265^{\mathrm{b}}$ & US-24 & 2011 & Freeville, NY & Potato \\
\hline 1246 & US-24 & 2011 & North Dakota & Potato \\
\hline 1247 & US-24 & 2011 & Caribou, ME & Potato \\
\hline 1219 & US-24 & 2011 & Hoople, ND & Unknown \\
\hline
\end{tabular}

a Isolates are stored in the laboratory of William Fry, Cornell University.

b Isolates were sequenced for each of the diagnostic regions to verify the presence of diagnostic single nucleotide polymorphisms. from other recently important U.S. lineages. Two methods were employed to achieve this. The first method utilized HRM analysis to differentiate US-23 from US-8, US-11, and US-24. Four HRM primer pairs were designed to differentiate lineages by targeting four separate genomic regions containing one, two, three, and nine polymorphisms. The second method utilized two differentially labeled LNA probes to differentiate lineage US-23 from three other recently important lineages (US-8, US-11, and US-24) in a multiplex qPCR.

\section{Materials and Methods}

Genome mining for informative loci. We mined genomes of Phytophthora bioinformatically to find diagnostic, polymorphic loci using the previously published strains T30-4 (Broad Institute); PIC99189 and 90128 (Raffaele et al. 2010); 13_a2 (Cooke et al. 2012); DDR7602, LBUS5, NL0743, P1362, P6096, P10127, P10650, P11633, P12204, P13527, P13626, and P17777 (Yoshida et al. 2013); and RS2009P1_us8, IN2009T1_us22, and BL2009P4_us23 (Martin et al. 2013). One strain of $P$. mirabilis was also included (Yoshida et al. 2013). Genomes of U.S. clonal lineages (isolates 1306_1, 1306_2, 1306_3, Pi-11-006, Pi-11-007, Pi-11-016, Pi-11-017, and Pi-11-019) were also resequenced at the University of California-Riverside using paired-end 50-bp Illumina sequencing. Libraries were prepared using a Paired End DNA Sample Prep Kit (Illumina), following the manufacturer's instructions. Libraries were then sequenced to an average depth of 27-fold using an Illumina HiSeq 2000. Base calling and quality filtering were done using the CASAVA 1.8.2 pipeline (Illumina).

The T30-4 P. infestans genome was used as a reference (Haas et al. 2009) to map reads using bowtie2 (Langmead and Salzberg 2012). Variants were called using SAMtools (Li et al. 2009), and the resulting VCF files (Danecek et al. 2011) were filtered on the first and third quartile of depth and a mapping quality between 20 and 50 using custom R scripts (https://github.com/knausb/vcfR). Custom R scripts were used to identify variants which were homozygous in all samples but segregated for each U.S. lineage and could be diagnostic loci for HRM and LNA assays.

To extract the genomic regions that contain diagnostic variants for each of the clonal lineages, the GATK FastaAlternateReferenceMaker tool (McKenna et al. 2010) was used. The regions extracted contained $500 \mathrm{bp}$ upstream and downstream of the variant site for all samples to allow for the design of HRM primers, LNA probes, and flanking primers. In the case where more than one variant was found within this $1-\mathrm{kb}$ region, the region was redefined as a region of $500 \mathrm{bp}$ upstream of the first variant (or the maximum possible, in the case where the variant was in proximity with the start of a supercontig) and $500 \mathrm{bp}$ downstream of the last variant (or the maximum possible, in case the variant was close to the end of a supercontig). Each of the polymorphic loci found represented candidate diagnostic loci.

$P$. infestans isolates used for diagnostic assays. Isolates were originally collected between 2008 and 2013 from across the United States and Canada (Table 1) and sent to the Fry lab for isolation and identification. Clonal lineages were previously determined in the Fry laboratory by microsatellite analysis (Fry et al. 2015; Lees et al. 2006). Isolates included 10 individuals from each of four United States clonal lineages (US-8, US-11, US-23, and US-24; $n=40$ ). Isolates were maintained in long-term storage in vials containing rye B agar (Caten and Jinks 1968). During short-term use, isolates were maintained on pea agar (Jaime-Garcia et al. 2000). Isolates were grown in pea broth (Goodwin et al. 1992) at $16^{\circ} \mathrm{C}$ for 5 to 10 days to obtain mycelia for DNA extractions. Mycelia were filtered from the broth, collected, and stored at $-20^{\circ} \mathrm{C}$ prior to extracting DNA. Extractions were done using a DNeasy Plant Mini Kit (Qiagen) according to the manufacturer's instructions.

HRM primer design and reaction information. Following genome mining for informative loci, four polymorphic regions were chosen as targets for the design of HRM primers. The four regions, named region $86,87,88$, and 92 , were selected for the presence of informative polymorphisms that would distinguish US-23 genotypes from non-US-23 genotypes. The close proximity of the polymorphisms 
was a prerequisite for HRM primer targets due to the need for HRM amplicons to be relatively short ( $\leq 110 \mathrm{bp}$ ). Primer pairs (Table 2 ) were designed using Primer-BLAST (National Center for Biotechnology Information; http://www.ncbi.nlm.nih.gov/tools/primer-blast/). Each primer pair was tested with the 40 isolates listed in Table 1, including 10 isolates from each of four clonal lineages.

All HRM and LNA qPCR assays were designed to meet the requirements of minimum information for publication of quantitative real-time experiments (MIQE guidelines; Bustin et al. 2009). PCR was done using a Bio-Rad CFX96 C1000 Touch thermal cycler (Bio-Rad). Samples were run in 96-well white Multiplate PCR plates and sealed with Microseal 'B' seals (Bio-Rad). Each reaction consisted of $10 \mu \mathrm{l}$ of Precision Melt Supermix (Bio-Rad), $1 \mu \mathrm{M}$ forward and reverse primers (Integrated DNA Technologies), and $2 \mathrm{ng}$ of template DNA brought to a final volume of $20 \mu \mathrm{l}$. Reactions were held at $95^{\circ} \mathrm{C}$ for $2 \mathrm{~min}$, followed by 45 cycles of $95^{\circ} \mathrm{C}$ for $10 \mathrm{~s}$ and $62^{\circ} \mathrm{C}$ for $30 \mathrm{~s}$, with fluorescence measured after each $62^{\circ} \mathrm{C}$ step. Following PCR target amplification, products were melted with the following program: $95^{\circ} \mathrm{C}$ for $30 \mathrm{~s}, 62^{\circ} \mathrm{C}$ for $1 \mathrm{~min}$, then $62^{\circ} \mathrm{C}$ for $10 \mathrm{~s}$, increasing to $95^{\circ} \mathrm{C}$ in $0.2^{\circ} \mathrm{C}$ increments, with fluorescence measured after each temperature increase. A control lacking template was included in each qPCR run. Cycle threshold $(\mathrm{Cq})$ values were determined using Bio-Rad CFX Manager software (version 3.0).

HRM analysis. HRM analysis was done using Bio-Rad Precision Melt Analysis software, version 1.2. Melt regions were automatically detected using the melt analysis software. For primers targeting regions 86 and 92 (primers Rgn86_1 and Rgn92_1), the default settings were used for cluster detection (temperature shift bar height $=0.20$, melt curve shape sensitivity $=50$, and $\mathrm{Tm}$ difference threshold $=0.15$ ). For primers targeting region 87 (primers Rgn87_1), shape sensitivity was adjusted from 50 to 37 and the Tm difference threshold was adjusted from 0.15 to 0.45 to achieve lineage separation. For primers targeting region 88 (primers Rgn88_1), the Tm difference threshold was adjusted from 0.15 to 0.1 to achieve lineage separation.

Sequencing diagnostic regions. Five isolates of each clonal lineage $(n=20)$ were sequenced bidirectionally to verify the presence of SNPs in each diagnostic region (region 86 , region 87 , region 88 , and region 92). Primers were designed to amplify a larger (approximately 400 to $800 \mathrm{bp}$ ) section of the region containing the SNPs because of the difficulty associated with sequencing small (approximately $60 \mathrm{bp}$ ) HRM products (Table 3). One primer pair per diagnostic region was designed using Primer-BLAST (http://www.ncbi.nlm. nih.gov/tools/primer-blast/; Table 3). Isolates chosen for sequencing are indicated in Table 1, and 250-bp sequences representing each lineage-region genotype were deposited in GenBank under accession numbers KT966883 to KT966900.

PCR assays were carried out in 50- $\mu$ l volumes containing $10 \mu \mathrm{l}$ of $5 \times$ green GoTaq reaction buffer (Promega), $1.25 \mathrm{U}$ of GoTaq DNA polymerase (Promega), $200 \mu \mathrm{M}$ dNTP, $400 \mathrm{nM}$ each forward and reverse primer, and $4 \mathrm{ng}$ of template DNA. PCR was done using a Bio-Rad C1000 Touch thermal cycler with the following reaction conditions: initial denaturation of $95^{\circ} \mathrm{C}$ for $3 \mathrm{~min}$, followed by 35 cycles of $95^{\circ} \mathrm{C}$ for $30 \mathrm{~s}, 60^{\circ} \mathrm{C}$ for $30 \mathrm{~s}$, and $72^{\circ} \mathrm{C}$ for $1 \mathrm{~min}$. Reactions were held at $72^{\circ} \mathrm{C}$ for $10 \mathrm{~min}$ for a final extension. PCR products were verified by gel electrophoresis prior to submission to the Cornell Institute for Biotechnology for sequencing. Sequences of each region were aligned using MEGA6 (Tamura et al. 2013) to verify the presence of each diagnostic SNP.

Further analysis was done on region 87 to determine the cause of unexpected HRM and sequencing results. Rgn87L_1 PCR products amplified from one US-8, US-11, US-23, and US-24 isolate were cloned using a TOPO TA Cloning Kit for Sequencing, according to the manufacturer's instructions (Invitrogen, Thermo Fisher Scientific). Competent Escherichia coli cells were transformed using a Mix \& Go E. coli Transformation Kit, according to the manufacturer's instructions (Zymo Research). Successful transformation was verified by colony PCR using primers T3 and T7 (TOPO TA Cloning Kit; Invitrogen) and the PCR protocol for sequencing previously described. Cloned PCR products were sequenced as previously described, and sequences were aligned using MEGA6 to determine genotypic differences among $P$. infestans lineages within region 87.

LNA probe and flanking primer design. The same polymorphisms targeted by the Rgn86_1 HRM primers, located in region 86 , were chosen as targets for LNA probes. Region 86 was chosen because of the presence of two polymorphisms within $4 \mathrm{bp}$ of each other which could be incorporated into the relatively short (11 bp) probe sequences. The probes were designed to distinguish between clonal lineage US-23 and non-US-23 lineages in a multiplex qPCR. Probes were designed by Integrated DNA Technologies. The first probe (PiLNA86PRhx), labeled with HEX at the $5^{\prime}$ end, was designed to be specific for US-23 alleles at the two SNP locations

Table 2. High-resolution melt primers for differentiating Phytophthora infestans clonal lineage US-23

\begin{tabular}{|c|c|c|c|c|c|}
\hline Primer pair & Primer sequence & Region & Location $(n)^{\mathbf{a}}$ & Amplicon size (bp) & Lineage groupings \\
\hline \multirow[t]{2}{*}{ Rgn86_1 } & Rgn86_1F: (5'-TTGCTGGATGGAGCTCGGAC-3') & Region 86 & $599,602(2)$ & 72 & (US-23) \\
\hline & Rgn86_1R: (5'-GGCAGTCACCGTGCGT-3') & & & & (US-8, US-11, US-24) \\
\hline \multirow[t]{3}{*}{ Rgn87_1 } & Rgn87_1F: (5'-GGAGGTCAGTGGAAACGGAA-3') & Region 87 & $501(1)$ & 63 & (US-23) \\
\hline & Rgn87_1R: (5'-GACGCTCGGTACAGTATCTGG-3') & $\ldots$ & $\ldots$ & $\ldots$ & (US-11) \\
\hline & & $\ldots$ & $\ldots$ & & (US-8, US-24) \\
\hline \multirow[t]{2}{*}{ Rgn88_1 } & Rgn88_1F: (5'-TGGACGCTATCATCCGACAG-3') & Region 88 & $501(1)$ & 60 & (US-23) \\
\hline & Rgn88_1R: (5'-GTCCACTTGACTGGACGTGT-3') & $\ldots$ & $\ldots$ & $\ldots$ & (US-8, US-11, US-24) \\
\hline \multirow[t]{2}{*}{ Rgn92_1 } & Rgn92_1F: (5'-GCCCACGAACGTTATCTATTTCT-3') & Region 92 & $501,525,552(3)$ & 110 & (US-23) \\
\hline & Rgn92_1R: (5'-ACTTAGCGTCCAAGTCAATGC-3') & $\ldots$ & $\ldots$ & $\ldots$ & (US-8, US-11, US-24) \\
\hline
\end{tabular}

Table 3. Primers used to amplify each diagnostic region for sequencing

\begin{tabular}{llcc}
\hline Primer pair & \multicolumn{1}{c}{ Primer sequence } & Region & Amplicon size (bp) \\
\hline Rgn86L_1 & Rgn86L_1F: (5'-ACAACCAAAAGGTGACGTGT-3') & 86 & 821 \\
& Rgn86L_1R: (5'-GCAAGTACAAGGCCCAGACA-3') & $\ldots$ & $\ldots$ \\
Rgn87L_1 & Rgn87L_1F: (5'-GAGGTATACCGCAAGCCGAA-3') & 87 & $\ldots$ \\
& Rgn87L_1R: $\left(5^{\prime}\right.$-TCGGCATCAACCAAGGTCTC-3') & $\ldots$ & $\ldots$ \\
Rgn88L_1 & Rgn88L_1F: (5'-TCGAAAGATTTCCGCGCTCA-3') & 88 & $\ldots$ \\
& Rgn88L_1R: $\left(5^{\prime}\right.$-ACTCTGCGGCAAATACACGA-3') & $\ldots$ & $\ldots$ \\
Rgn92L_1 & Rgn92L_1F: (5'-TTCCCCTCTTTGTCGTCACAAT-3') & 92 & $\ldots$ \\
& Rgn92L_1R: $\left(5^{\prime}\right.$-TTGTTGCCCTTCTCCACGGT-3') & $\ldots$ & \\
\hline
\end{tabular}


(Table 4). The second probe (PiLNA86PRfm), labeled with FAM at the $5^{\prime}$ end, was designed to be specific for non-US-23 alleles at the two SNP locations (Table 4). Both probes included Iowa Black FQ quencher at the $3^{\prime}$ end. Flanking primers (PiLNA_2F and PiLNA_2R) designed to amplify a 231-bp section of region 86 containing the two diagnostic SNPs, were designed using Primer-BLAST (http://www.ncbi.nlm.nih.gov/tools/primer-blast/, Table 4).

LNA assay and optimization. LNA reactions were run in 96-well white Multiplate PCR plates (Bio-Rad) and sealed with Microseal ' $\mathrm{B}$ ' seals (Bio-Rad). Reactions were carried out in $20-\mu$ l volumes containing $10 \mu \mathrm{l}$ of iQ supermix ( $1 \times$ final concentration; Bio-Rad), $500 \mathrm{nM}$ each forward and reverse flanking primer, $250 \mathrm{nM}$ each LNA probe (PiLNA86PRfm and PiLNA86PRhx), and $3 \mathrm{ng}$ of

Table 4. Locked nucleic acid (LNA) probes and flanking primers for differentiating US-23 from non-US-23 isolates

\begin{tabular}{ll}
\hline Probe or primer & \multicolumn{1}{c}{ Sequence } \\
\hline LNA probe & \\
PiLNA86PRhx & 5HEX/CGT +C +GC +C GAGA/3IaBkFQ \\
PiLNA86PRfm & 56FAM/CG+T +T +GC +T +GAGA/3IaBkFQ \\
Flanking primer & \\
PiLNA86_2F & 5'-TGCCCCAGTGGACAACAAAG-3' \\
PiLNA86_2R & $5^{\prime}$-CATCCTGGTCCCAACCAACA-3' \\
\hline
\end{tabular}

${ }^{a}$ Flanking primers amplified a 231-bp section of region 86.

Table 5. Differentiation of Phytophthora infestans clonal lineages following a multiplex quantitative polymerase chain reaction run with two differentially labeled locked nucleic acid (LNA) probes ${ }^{\mathrm{a}}$

\begin{tabular}{|c|c|c|c|c|c|}
\hline Isolate & Lineage & Fluorophore & $\mathbf{C q}$ & Fluorophore & $\mathbf{C q}$ \\
\hline 112322 & US-23 & HEX & 24.29 & FAM & N/A \\
\hline 122312 & US-23 & HEX & 23.71 & FAM & N/A \\
\hline 122322 & US-23 & HEX & 23.24 & FAM & N/A \\
\hline 122329 & US-23 & HEX & 23.33 & FAM & N/A \\
\hline 132317 & US-23 & HEX & 24.08 & FAM & N/A \\
\hline 0982 & US-8 & HEX & N/A & FAM & 22.62 \\
\hline 1083 & US-8 & HEX & N/A & FAM & 21.83 \\
\hline 1087 & US-8 & HEX & N/A & FAM & 23.05 \\
\hline 1185 & US-8 & HEX & N/A & FAM & 22.76 \\
\hline 1381 & US-8 & HEX & N/A & FAM & 22.79 \\
\hline 11111 & US-11 & HEX & N/A & FAM & 21.55 \\
\hline 11114 & US-11 & HEX & N/A & FAM & 20.45 \\
\hline 11115 & US-11 & HEX & N/A & FAM & 19.28 \\
\hline 11117 & US-11 & HEX & N/A & FAM & 21.65 \\
\hline 13111 & US-11 & HEX & N/A & FAM & 23.55 \\
\hline 1249 & US-24 & HEX & N/A & FAM & 23.59 \\
\hline 1250 & US-24 & HEX & N/A & FAM & 22.62 \\
\hline 1273 & US-24 & HEX & N/A & FAM & 22.42 \\
\hline 1339 & US-24 & HEX & N/A & FAM & 23.38 \\
\hline 1345 & US-24 & HEX & N/A & FAM & 23.19 \\
\hline US110047b & US-23 & HEX & + & FAM & - \\
\hline $\mathrm{MN}-10-1^{\mathrm{b}}$ & US-23 & HEX & + & FAM & - \\
\hline US100024 & US-8 & HEX & - & FAM & + \\
\hline CA-10-1 ${ }^{b}$ & US-8 & HEX & - & FAM & + \\
\hline US110160 & US-11 & HEX & - & FAM & + \\
\hline AK-10-1b & US-11 & HEX & - & FAM & + \\
\hline US100059 & US-24 & HEX & - & FAM & + \\
\hline ND-10-1 ${ }^{b}$ & US-24 & HEX & - & FAM & + \\
\hline $\mathrm{T}-30-4^{\mathrm{b}}$ & NA & HEX & - & FAM & + \\
\hline \multicolumn{6}{|c|}{$\begin{array}{l}\text { a The HEX-labeled probe (PiLNA86PRhx) specifically anneals to the US-23 } \\
\text { target sequence due to two single nucleotide polymorphisms (SNPs) located } \\
\text { within the region } 86 \text { portion (PITG } 11903 \text { gene) of supercontig_1.21. The } \\
\text { FAM-labeled probe (PiLNA86PRfm) specifically anneals to the non-US- } \\
23 \text { target sequence containing the alternate alleles at the two SNP loci. } \\
\mathrm{Cq}=\text { cycle threshold and N/A = not available. } \\
\text { b Isolates were independently tested at the United States Department of Agri- } \\
\text { culture-Agricultural Research Service HCRL, Corvallis, OR. Only qualita- } \\
\text { tive data were reported for these tests. }\end{array}$} \\
\hline
\end{tabular}

template DNA ( $3 \mu \mathrm{l}$ of template DNA at $1 \mathrm{ng} / \mu \mathrm{l}$, unless otherwise noted). Several LNA reactions were run at varying annealing temperatures to optimize the reaction for lineage separation. Six annealing temperatures were tested, ranging from 60.0 to $67.0^{\circ} \mathrm{C}$. In each test of optimal annealing temperature, one isolate from each clonal lineage was tested, along with a control which lacked template. LNA reactions used a Bio-Rad CFX96 C1000 Touch thermal cycler and the following reaction conditions: initial denaturation of $95.0^{\circ} \mathrm{C}$ for $3 \mathrm{~min}$, followed by 35 cycles of $95.0^{\circ} \mathrm{C}$ for $10 \mathrm{~s}$ and $65.8^{\circ} \mathrm{C}$ (or varied annealing temperatures during optimization tests) for $45 \mathrm{~s}$, with fluorescence measured after each $65.8^{\circ} \mathrm{C}$ step. Each qPCR run included a control which lacked template. Cq values were determined using Bio-Rad CFX Manager software (version 3.0).

LNA probe sensitivity and specificity. Lineage specificity. Following reaction optimization, LNA probes were tested for lineage specificity with five isolates from each of four lineages (US-8, US11 , US-23, and US-24; $n=20$; Table 5). Each isolate was tested in a separate reaction, as previously described.

In addition to specificity, assay sensitivity was determined by running 10-fold dilution series of P. infestans DNA from 3,000 to 0.3 $\mathrm{pg} / \mathrm{reaction}$. Three US-23 dilution series were tested (isolates 122312,122322 , and 122329) along with three non-US-23 isolates (1087 [US-8], 13111 [US-11], and 1339 [US-24]). The limit of detection was defined as the lowest concentration of template DNA that still resulted in detection by the target probe. Standard curves for each probe were generated using Microsoft Excel (Microsoft).

Mixed-lineage reactions were also done at varying DNA concentrations to determine the effect on each LNA probe's ability to separate lineages. A total of three two-lineage combinations were tested (US-23 + US-8, US-23 + US-11, and US-23 + US-24), each at eight DNA concentration combinations (Table 6). Reaction mixes were prepared as previously described, with the exception of $6 \mu l$ of template DNA ( $3 \mu \mathrm{l}$ of US-23 DNA [variable concentration] and $3 \mu \mathrm{l}$ of non-US-23 DNA [variable concentration]) being added to each reaction well.

The LNA assay was independently tested in Corvallis, OR using nine isolates of $P$. infestans representing four lineages. Isolates included two individuals from each of the US-8, US-11, US-23, and

Table 6. Limit of detection of the multiplex locked nucleic acid probe assay and threshold cycle $(\mathrm{Cq})$ values for mixed-lineage samples run at varying DNA concentrations

\begin{tabular}{|c|c|c|c|}
\hline \multicolumn{2}{|c|}{$\begin{array}{l}\text { Phytophthora infestans } \\
\text { template DNA (pg) }\end{array}$} & \multicolumn{2}{|c|}{$\mathrm{Cq} \pm$ standard deviation $^{\mathrm{a}}$} \\
\hline $\begin{array}{l}\text { US-23 } \\
\text { lineage }\end{array}$ & $\begin{array}{c}\text { Non-US-23 } \\
\text { lineage }^{b}\end{array}$ & $\begin{array}{c}\text { HEX } \\
\text { fluorophore }\end{array}$ & $\begin{array}{c}\text { FAM } \\
\text { fluorophore }\end{array}$ \\
\hline 3,000 & None & $22.71 \pm 0.24$ & nd \\
\hline 300 & None & $26.24 \pm 0.21$ & nd \\
\hline 30 & None & $29.50 \pm 0.22$ & nd \\
\hline 3 & None & $33.16 \pm 0.46$ & nd \\
\hline None & 3,000 & nd & $21.73 \pm 0.59$ \\
\hline None & 300 & nd & $24.97 \pm 0.57$ \\
\hline None & 30 & nd & $28.80 \pm 0.23$ \\
\hline None & 3 & nd & $31.94 \pm 1.00$ \\
\hline 3,000 & 3,000 & $21.46 \pm 0.20$ & $21.59 \pm 0.25$ \\
\hline 300 & 300 & $24.98 \pm 0.98$ & $25.28 \pm 0.17$ \\
\hline 30 & 30 & $28.67 \pm 0.10$ & $29.11 \pm 0.25$ \\
\hline 3 & 3 & $32.38 \pm 0.73$ & $32.85 \pm 0.28$ \\
\hline 3,000 & 30 & $21.58 \pm 0.51$ & nd \\
\hline 300 & 30 & $25.23 \pm 0.54$ & nd \\
\hline 30 & 3,000 & nd & $21.46 \pm 0.22$ \\
\hline 30 & 300 & nd & $24.82 \pm 0.63$ \\
\hline
\end{tabular}

a Values are the mean of three biological replicates; nd = not detected.

b Non-US-23 lineages included one isolate of each US-8, US-11, and US-24 lineages. Isolates were tested independently and together represented three biological replicates of non-US-23 isolates. 
US-24 lineages, along with an additional strain (T30-4) which has served as a reference strain in numerous other studies (Table 5). The LNA assay was conducted as previously described.

\section{Results}

Genome mining for informative loci. From a panel of 28 isolates, a total of 407,014 variants were called. From these, 246 homozygous variants which were diagnostic for U.S. clonal lineages were observed. Among those, 43 SNPs were putatively diagnostic for lineage US-23. Four regions within supercontig_1.21 (regions 86, 87, 88, and 92), each containing at least one US-23 diagnostic SNP, were chosen for the development of lineage-diagnostic assays. Region 86 had two polymorphisms diagnostic for US-23: two T $\rightarrow$ C substitutions at positions 876,626 and 876,629 . This portion of supercontig_1.21 includes the gene PITG_11903 (conserved hypothetical protein). Region 87 had one polymorphism diagnostic for US-23: a $\mathrm{G} \rightarrow$ A substitution at position 882,228. This portion of supercontig_1.21 did not align with any known annotated gene sequences when searched against the BLAST nr database, the Broad Institute $P$. infestans genome (http://www.broadinstitute.org/annotation/ genome/phytophthora_infestans/MultiHome.html), and FungiDB (Stajich et al. 2012). Region 88 had one polymorphism diagnostic for US-23: a C $\rightarrow$ T substitution at position 883,272 . This portion of supercontig_1.21 also did not align with any known annotated gene sequences when searched against the previously mentioned databases. Region 92 had three polymorphisms diagnostic for US-23: a $\mathrm{T} \rightarrow \mathrm{C}$ substitution at position 933,678, a $\mathrm{C} \rightarrow \mathrm{T}$ substitution at position 933,702, and a $\mathrm{C} \rightarrow$ T substitution at position 933,729. This portion of supercontig_1.21 includes the gene PITG_11913 (heat shock cognate 70-kDa protein).

HRM analysis. Primers Rgn86_1 HRM. Primers Rgn86_1 were designed to amplify a 72-bp sequence of region 86 . This region of the $P$. infestans genome contained two US-23 diagnostic SNPs based on results from genome mining for informative loci. Using Bio-Rad Precision Melt Analysis software and default settings for cluster detection, these primers differentiated all 10 US-23 isolates from all 30 non-US-23 isolates (US-8, $n=10$; US- $11 ; n=10$; and US-24, $n=10$; Fig. 1). The average Tm for US- 23 isolates was $85.5^{\circ} \mathrm{C}$. The average Tm for non-US-23 isolates was $84.6^{\circ} \mathrm{C}$ (Table 7).

We also checked the sequence of the region amplified in this HRM assay by amplifying the region using primer pair Rgn86L_1, which generates a 821-bp sequence containing the HRM amplicon. Five isolates from each of the four lineages $(n=20)$ were sequenced bidirectionally to verify the presence of diagnostic SNPs. Following sequence alignment, both SNPs were present in the expected locations for all 20 isolates. Besides the two expected SNPs, all 20 genotypes were identical for the region $86 \mathrm{HRM}$ amplicon (GenBank accession numbers KT966883 to KT966886).

Primers Rgn87_1 HRM. Primers Rgn87_1 were designed to amplify a 63-bp sequence of region 87 . This region of the $P$. infestans genome contained one US-23 diagnostic SNP based on results from genome mining for informative loci. Using Bio-Rad Precision Melt Analysis software and manual adjustments for cluster detection, these primers generated three clusters following HRM analysis: cluster $1=\mathrm{US}-8(n=10)$ and US-24 $(n=10)$, cluster $2=\mathrm{US}-11(n=10)$, and cluster $3=\mathrm{US}-23(n=10)$. The average Tm for US-23 isolates was $77.2^{\circ} \mathrm{C}$. The average Tm for US-8 and US-24 isolates was $76.8^{\circ} \mathrm{C}$. The average Tm for US- 11 isolates was $76.6^{\circ} \mathrm{C}$ (Table 7).

We also checked the sequence of the region amplified in this HRM assay by amplifying the region using primer pair Rgn87L_1, which generates a 413-bp sequence containing the HRM amplicon. Five isolates from each of the four lineages $(n=20)$ were sequenced bidirectionally to verify the presence of the diagnostic SNP. Following sequence alignment, the known US-23 diagnostic SNP was present in the expected location. However, there was also an unexpected 2-bp deletion in non-US-23 isolates 4 bp away from the known SNP and within the HRM amplicon (Fig. 2). Some of the sequence files for non-US-23 isolates showed double peaks immediately following the location of the 2-bp deletion in both the forward and reverse directions. This indicated that these isolates were likely heterozygous for the 2-bp deletion.
In order to definitively understand the basis for the unexpected Rgn87_1 HRM results, Rgn87L_1 PCR products amplified from one US-8, US-11, US-23, and US-24 isolate were cloned into a vector, and two successfully transformed $E$. coli colonies from each isolate were sequenced to determine the genotype of each lineage (GenBank accession numbers KT966887 to KT966892). Following cloning, sequencing alignments revealed nine polymorphisms among the four clonal lineages within the 63-bp HRM amplicon. A heterozygous 2-bp deletion was identified in both US-24 and US-8 individuals. The US-11 individual was homozygous for this 2-bp deletion, while the US-23 individual was homozygous for GC at this locus. Additionally, the US-24 individual showed two heterozygous SNPs within the HRM amplicon. The US-23 diagnostic SNP identified by genome mining was present in the expected location (Fig. 2).

Primers Rgn88_1 HRM. Primers Rgn88_1 were designed to amplify a 60 -bp sequence of region 88 . This region of the $P$. infestans genome contained one US-23 diagnostic SNP based on results from genome mining for informative loci. Using Bio-Rad Precision Melt Analysis software and manual adjustments for cluster detection, these primers differentiated all 10 US-23 isolates from all 30 non-US-23 isolates (US-8, $n=10$; US-11; $n=10$; and US-24, $n=10$; Fig. 1 ). The average Tm for US-23 isolates was $81.8^{\circ} \mathrm{C}$. The average $\mathrm{Tm}$ for non-US-23 isolates was $82.0^{\circ} \mathrm{C}$ (Table 7). The controls lacking template failed to produce an amplification curve in any of the HRM qPCR runs.

We also checked the sequence of the region amplified in this HRM assay by amplifying the region using primer pair Rgn88L_1, which generates a 381-bp sequence containing the HRM amplicon. Five isolates from each of the four lineages $(n=20)$ were sequenced bidirectionally to verify the presence of the diagnostic SNP. Following sequence alignment, the SNP was present in the expected location for all 20 isolates. Besides the expected SNP, all 20 genotypes were identical for the region 88 HRM amplicon (GenBank accession numbers KT966893 to KT966896).

Primers Rgn92_1 HRM. Primers Rgn92_1 were designed to amplify a 110-bp sequence of region 92 . This region of the $P$. infestans genome contained three US-23 diagnostic SNPs based on results from genome mining for informative loci. Using Bio-Rad Precision Melt Analysis software and default settings for cluster detection, these primers differentiated all 10 US-23 isolates from all 30 nonUS-23 isolates (US-8, $n=10$; US-11; $n=10$; and US-24, $n=10$; Fig. 1). The average Tm for US-23 isolates was $85.3^{\circ} \mathrm{C}$. The average $\mathrm{Tm}$ for non-US-23 isolates was $86.0^{\circ} \mathrm{C}$ (Table 7).

We also checked the sequence of the region amplified in this HRM assay by amplifying the region using primer pair Rgn92L_1, which generates a 406-bp sequence containing the HRM amplicon. Five isolates from each of the four lineages $(n=20)$ were sequenced bidirectionally to verify the presence of diagnostic SNPs. Following sequence alignment, all three SNPs were present in the expected location for all 20 isolates. Besides the three expected SNPs, all 20 genotypes were identical for the region $92 \mathrm{HRM}$ amplicon (GenBank accession numbers KT966897 to KT966900).

Lineage differentiation using LNA probes. Two differentially labeled LNA probes, PiLNA86hx and PiLNA86fm, were designed to target a portion of region 86 and differentiate US-23 isolates from non-US-23 isolates in a multiplex qPCR. At an optimized annealing temperature of $65.8^{\circ} \mathrm{C}$, the LNA probes separated 5 US-23 isolates from 15 non-US-23 isolates (Fig. 3; Table 5). Perfect discrimination

Table 7. Average melting temperature of each high-resolution melt amplicon by clonal lineage

\begin{tabular}{lcccc}
\hline & \multicolumn{4}{c}{ Melt temperature \pm one standard deviation $\left({ }^{\circ} \mathbf{C}\right)^{\mathbf{a}}$} \\
\cline { 2 - 5 } HRM primer pair & US-23 & US-11 & US-8 & US-24 \\
\hline Rgn86_1 & $85.5 \pm 0.1$ & $84.6 \pm 0.0$ & $84.6 \pm 0.0$ & $84.6 \pm 0.1$ \\
Rgn87_1 & $77.2 \pm 0.0$ & $76.6 \pm 0.1$ & $76.9 \pm 0.2$ & $76.7 \pm 0.1$ \\
Rgn88_1 & $81.8 \pm 0.0$ & $82.0 \pm 0.1$ & $82.0 \pm 0.1$ & $82.0 \pm 0.0$ \\
Rgn92_1 & $85.3 \pm 0.1$ & $86.0 \pm 0.1$ & $86.0 \pm 0.0$ & $86.0 \pm 0.2$ \\
\hline
\end{tabular}

a Phytophthora infestans clonal lineage average melting temperature. Each value represents the mean of 10 biological replicates. 

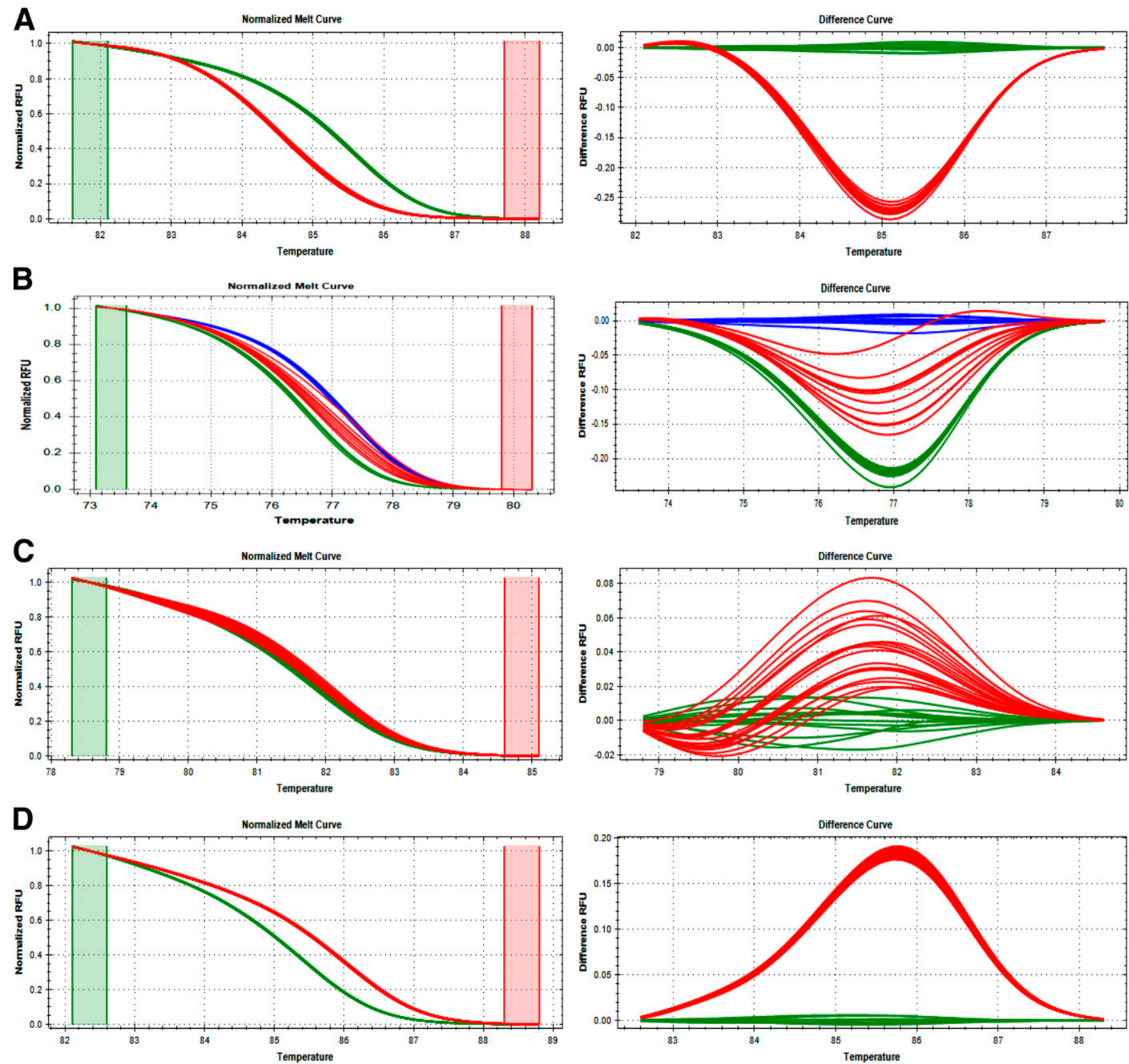

Fig. 1. Phytophthora infestans clonal lineage differentiation following high-resolution melt analysis. A, Primers Rgn86_1 separate US-23 genotypes (green bands) from US-8, US11, and US-24 genotypes (red bands) based on two polymorphisms. B, Primers Rgn87_1 separate US-23 genotypes (blue bands) from US-8 and US-24 genotypes (red bands), and US-11 genotypes (green bands) based on nine polymorphisms. C, Primers Rgn88_1 separate US-23 genotypes (green bands) from US-8, US-11, and US-24 genotypes (red bands) based on one polymorphism. D, Primers Rgn92_1 separate US-23 genotypes (green bands) from US-8, US-11, and US-24 genotypes (red bands) based on three polymorphisms. Left panel: normalized melt curves with vertical bars indicating premelt range (left, green bar) and postmelt range (right, red bar). Right panel: difference curves.

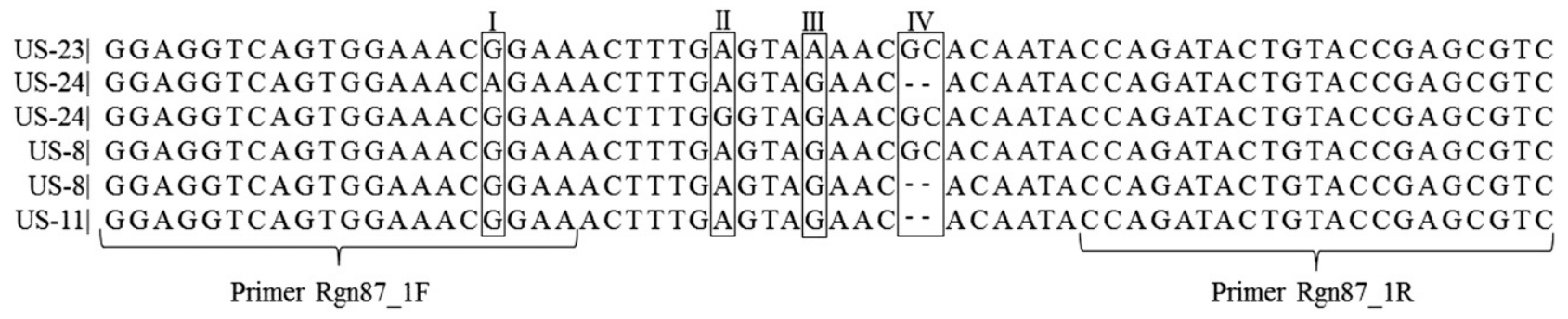

Fig. 2. Sequencing alignments following cloning of the high-resolution melt target portion of region 87. Columns I and II: US-24 contained two heterozygous single nucleotide polymorphisms (SNPs), one of which was located within the forward primer sequence. Column III: The US-23 diagnostic SNP originally identified through genome mining. Column IV: A 2-bp deletion, heterozygous in US-8 and US-24 individuals and homozygous in the US-11 individual. 
was observed between US-23 isolates, detected by the HEX fluorophore in the PiLNA86hx probe, and non-US-23 isolates, detected by the FAM fluorophore in the PiLNA86fm probe (Fig. 3; Table 5). Cq values for US-23 isolates ranged from 23.24 to 24.49 with the HEX fluorophore. US-23 isolates did not generate Cq values with the FAM fluorophore. Non-US-23 isolates generated $\mathrm{Cq}$ values of 19.28 to 23.59 with the FAM fluorophore but did not generate $\mathrm{Cq}$ values with the HEX fluorophore (Table 5). To ensure reproducibility, the LNA assay was independently tested in the coauthors' laboratories at Oregon State and Cornell University. The LNA assay differentiated two US-23 individuals from seven non-US-23 individuals (US-8, $n=2$,
US-11, $n=2$, US-24, $n=2$, and strain T30-4; Table 5). The controls lacking template failed to produce an amplification curve in any LNA qPCR.

LNA assay limit of detection. Ten-fold serial dilutions of $P$. infestans DNA were tested to determine the limit of detection of the LNA assay. Starting DNA concentrations ranged from 3,000 to $0.3 \mathrm{pg}$. In each of the three biological replicates, 3 pg of DNA was the minimum amount of DNA able to be detected by each LNA probe (Table 6; Fig. 4).

Testing LNA assay performance with mixed-lineage DNA samples. The multiplex LNA assay was tested with mixed-lineage DNA samples to determine whether assay performance would be

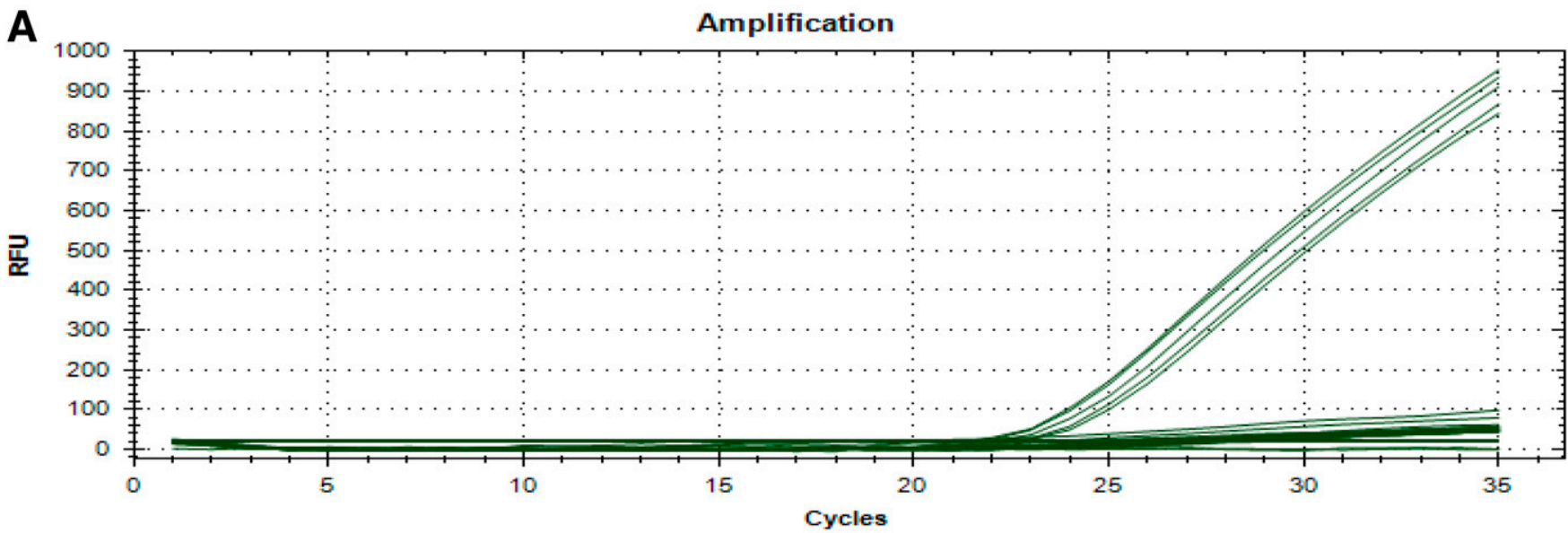

B

Amplification

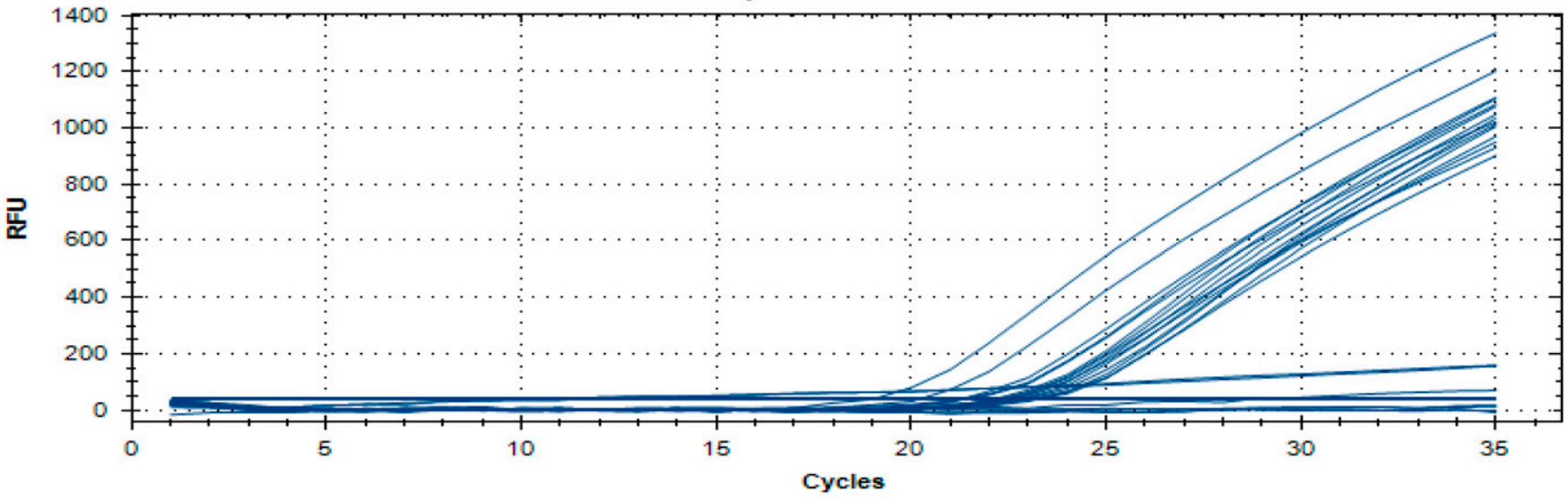

C

Amplification

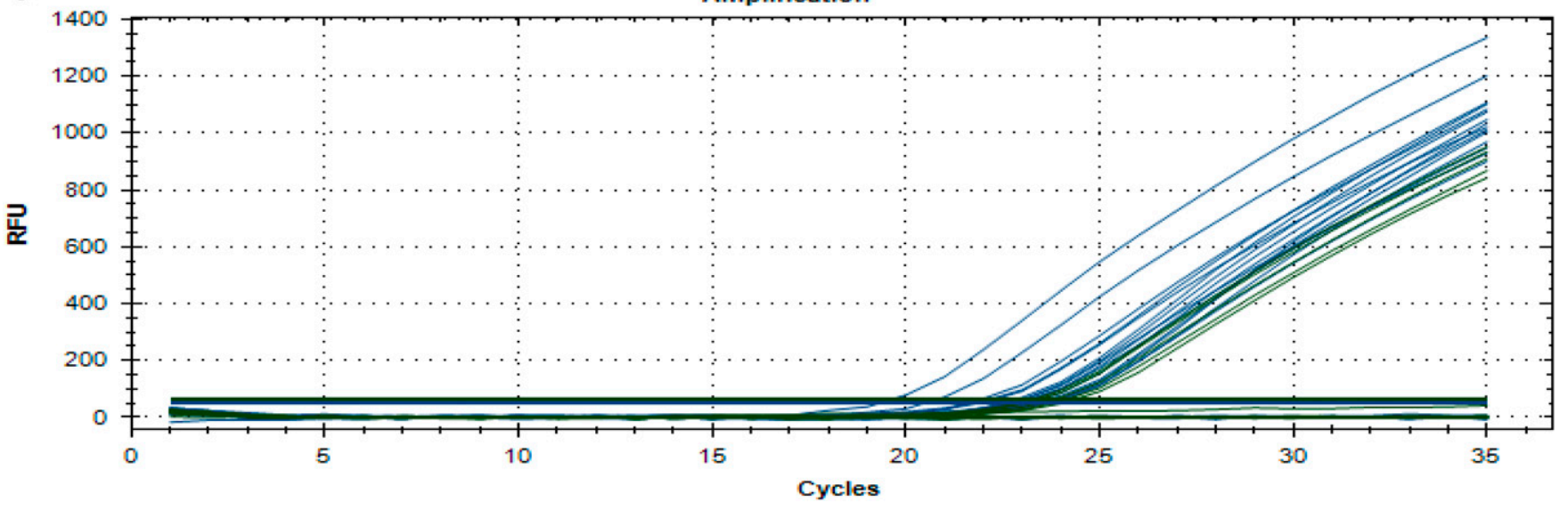

Fig. 3. Phytophthora infestans clonal lineage differentiation using locked nucleic acid probes. A, The HEX fluorophore (probe PiLNA86PRhx) generates exponential growth curves for isolates of lineage US-23 $(n=5)$ only. Lineage differentiation is due to specific binding of the PiLNA86PRhx LNA probe to the US-23 target sequence containing two SNPs. B, The FAM fluorophore (probe PiLNA86PRfm) generates exponential growth curves for isolates not belonging to lineage US-23 ( $n=15)$ (US-8, US-11, and US-24). C, Simultaneous detection of both HEX and FAM fluorophores generates exponential growth curves for both US-23 and non-US-23 isolates. Lineages can be distinguished by green curves (HEX, US-23) and blue curves (FAM, non-US-23). 
affected. To each reaction well, $6 \mu l$ of DNA ( $3 \mu l$ of US-23 and $3 \mu 1$ of non-US-23) were added at varying concentrations, and reactions were run as previously described (Table 6). When concentrations of US-23 and non-US-23 DNA were equal, the LNA assay performed similarly whether running a mixed-lineage or single-lineage reaction (Table 6). During a single-lineage reaction, either the HEX or FAM fluorophore was detected per well, depending on the lineage of the template DNA added to that well. During a mixed-lineage reaction, both HEX and FAM fluorophores were detected in each well, and the $\mathrm{Cq}$ values of each were comparable with those observed during the single-lineage limit of detection reactions (Tables 5 and 6). When concentrations of US-23 and non-US23 DNA differed by at least one order of magnitude, the assay only detected the DNA at the higher concentration (Table 6).

\section{Discussion}

The goal of this project was to develop molecular diagnostic assays capable of distinguishing isolates of $P$. infestans belonging to lineage US-23 from other lineages. Clonal lineages generally have conserved phenotypic characteristics such as host preference and fungicide sensitivity (Danies et al. 2013; Hu et al. 2012; Legard et al. 1995). Therefore, clonal lineages must be rapidly and accurately diagnosed in order for late blight management decisions to be as informed as possible. US-23 has been the predominant clonal lineage in the United States for several consecutive years (Fry et al. 2015). These assays could offer more rapid and accessible alternatives to diagnosing $P$. infestans lineage US-23 or could be used as a quick preliminary screening method.

Following the alignment of 27 whole $P$. infestans genomes and identification of 407,014 variants, 43 SNPs were identified as putatively diagnostic for lineage US-23. Four distinct regions located on supercontig_1.21 were chosen as targets for the development of diagnostic assays. Four HRM primer pairs targeted two, nine, one, and three polymorphisms within regions $86,87,88$, and 92 , respectively. Primers were designed to distinguish $P$. infestans US-23 genotypes from three recently occurring non-US-23 genotypes. The first primer pair, Rgn86_1, targeted two SNPs within 4 bp to differentiate US-23 from non-US-23 isolates. These primers produced consistent difference curves (Fig. 1B). They also produced the greatest Tm difference $\left(0.9 \pm 0.1^{\circ} \mathrm{C}\right)$ between the two genotypes of the four HRM primer pairs (Table 7), indicating that the Rgn86_1 HRM primers would provide a very reliable assay for diagnosing $P$. infestans lineage US-23. The second HRM primer pair, Rgn87_1, produced the surprising result of identifying three groups among the four lineages tested: US-23, US-11, and US-8 plus US-24 (Fig. 1D). This result was surprising because, following genome alignments and identification of informative SNPs, only one SNP diagnostic for US-23 was identified in region 87. However, following HRM analysis and subsequent cloning and sequencing of the diagnostic portion of region 87, there were nine polymorphisms identified (Fig. 2). Based on sequencing results, the Rgn87_1 HRM assay should have distinguished between US-24 and US- 8 individuals, which differed by two heterozygous SNPs (Fig. 2). It is possible that the heterozygous state of these SNPs, along with the heterozygous 2-bp deletion present in both US-8 and US-24 individuals, reduced the ability of HRM analysis to differentiate the two genotypes. The Rgn87_1 HRM assay provides more information than the other three HRM assays through the additional ability to distinguish US-11 from the other genotypes. However, a higher degree of variability in the difference curves for US-11, US-8, and US-24 genotypes (Fig. 1), along with relatively small $\mathrm{Tm}$ differences between some of the genotypes (US-11 and US-24 Tm difference $0.1 \pm 0.1{ }^{\circ} \mathrm{C}$ and US-23 and US-8 Tm difference $0.3 \pm 0.2^{\circ} \mathrm{C}$; Table 7) may make this assay slightly less reliable than Rgn86_1 and Rgn92_1 for assessing whether a sample belongs to the US-23 lineage. The third primer pair, Rgn88_1, distinguished US-23 from non-US-23 lineages based on one SNP. Although genotype discrimination was consistent, these primers showed the greatest degree of variability in difference curves (Fig. 1A). These primers also produced the smallest Tm difference $\left(0.2 \pm 0.1^{\circ} \mathrm{C}\right)$ between the two genotypes (Table 7$)$. These primers demonstrate that $P$. infestans lineages can be distinguished based on one SNP. However, among the assays described in this study, the Rgn88_1 HRM primers do not provide the most robust lineage diagnostic assay. The fourth HRM primer pair, Rgn92_1, targeted three SNPs to differentiate US-23s from non-US-23s. These primers also produced very consistent difference curves and a relatively high Tm difference $\left(0.7 \pm 0.2^{\circ} \mathrm{C}\right)$ between US-23 and non-US-23 genotypes (Fig. 1C; Table 7). Like the Rgn86_1 primers, the Rgn92_1 primers would provide a very reliable assay for diagnosing lineage US-23.

In addition to HRM, an LNA assay was developed to target two SNPs within 4 bp of each other in region 86 . This was chosen as the target for the design of LNA probes because of the opportunity to incorporate both SNPs into the probe target sequences, which

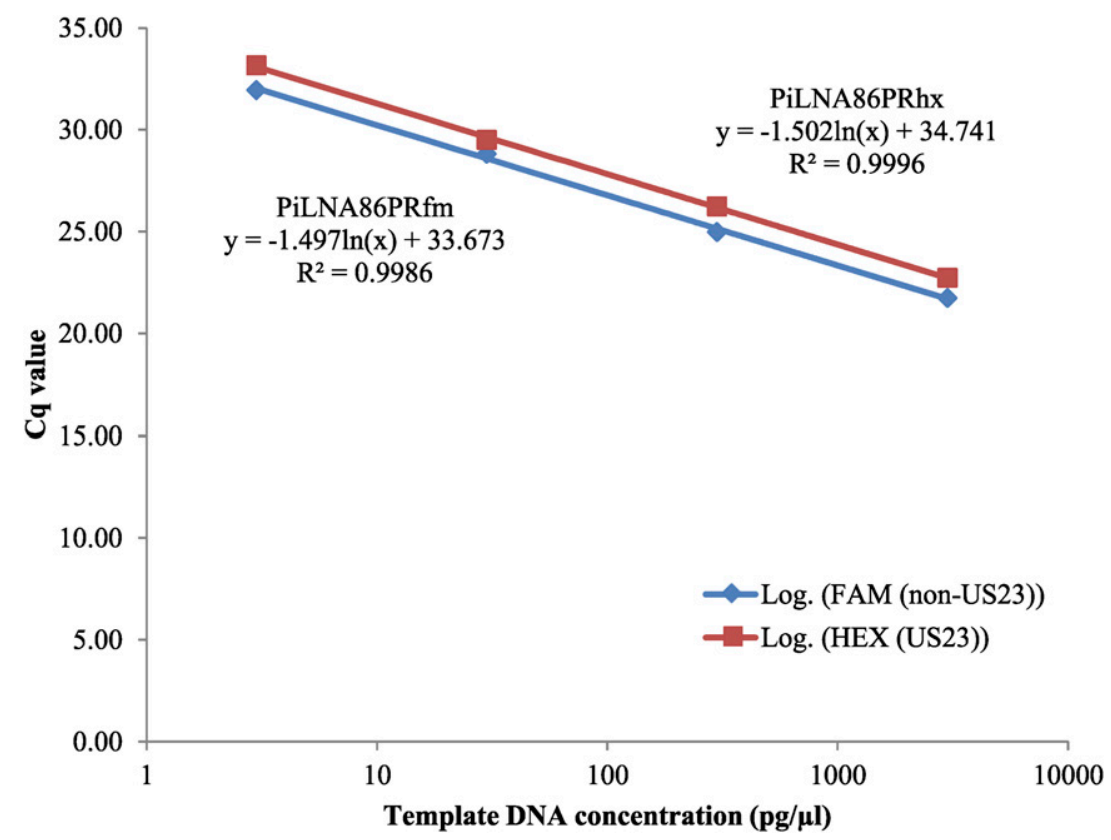

Fig. 4. Standard curves generated using 10-fold serial dilutions of Phytophthora infestans DNA. Concentrations ranged from 3,000 to 3 pg of DNA per reaction. Curves represent the mean of three biological replicates (probe PiLNA86PRhx, three US-23 isolates; probe PiLNA86PRfm, one of each US-8, US-11, and US-24 isolates). 
would offer increased discriminatory potential over the targeting of one SNP. This is because the incorporation of LNA into DNA probes increases the Tm of the resulting DNA duplex (Braasch and Corey 2001). As a result, a nucleotide mismatch at the site of an LNA dramatically decreases the Tm of the resulting DNA duplex. This is the basis for the enhanced specificity offered by LNA probes. The US23-specific probe PiLNA86PRhx had a theoretical Tm of $64^{\circ} \mathrm{C}$ when perfectly matched to its target DNA sequence. With one mismatch at the site of an LNA, the theoretical Tm was reduced by $16^{\circ} \mathrm{C}$, and two mismatches reduced the theoretical $\mathrm{Tm}$ by $40^{\circ} \mathrm{C}$. The probe PiLNA86PRfm, which targeted non-US-23 genotypes, had a theoretical $\mathrm{Tm}$ of $64.5^{\circ} \mathrm{C}$ when perfectly matched to its target DNA sequence. Theoretical Tm were reduced by 8.7 and $22^{\circ} \mathrm{C}$ with one or two LNA mismatches, respectively. This means that only perfect matches between probe and complementary sequences are able to hybridize at the optimized temperature of $65.8^{\circ} \mathrm{C}$ and, therefore, only the target genotype is detected. Although not empirically validated in this study, these theoretical Tm difference projections indicate the robustness of the LNA probes for distinguishing between the US-23 and non-US-23 genotypes.

The assays developed in this study could have immediate utility in plant disease diagnostic laboratories. Identifying which lineage of $P$. infestans a given isolate belongs to is crucial for informing late blight management decisions, and should be considered a necessary measure by plant pathology diagnostic laboratories tasked with diagnosing late blight samples. Currently, $P$. infestans lineages are identified by SSR analysis (Hu et al. 2012), which has the advantage of providing more information than SNP-based diagnostics, including the ability to identify novel genotypes. However, SSR analysis requires a DNA fragment analyzer, which is not available in most plant pathology diagnostic laboratories, unlike the qPCR machine required for the SNP-based US-23 assays described here. Results could be obtained more quickly with SNP-based diagnostic assays versus SSR analysis, especially if samples could be analyzed locally rather than being shipped to a central laboratory for analysis. Finally, costs per sample can be significantly less with SNP-based diagnostics compared with microsatellite analysis. Each LNA reaction costs approximately $\$ 1$ to $\$ 2$ per sample depending on the scale of reagent procurement. HRM analysis costs less than $\$ 1.00$ per reaction, not including the price of the HRM analysis software (about $\$ 3,300$; Bio-Rad Precision Melt Analysis software). SSR analysis is generally more expensive than both LNA and HRM analysis on a persample basis. This is especially true when the assays are used in a lineage diagnostic application, where relatively few samples are analyzed at a given time. At Cornell University, fragment analysis is done on a 96-well plate basis. Each plate costs $\$ 42.50$ for internal users and $\$ 68.00$ for external users. This means each sample costs between \$0.44 (96 samples) and \$42.50 (1 sample) for internal users and between $\$ 0.71$ (96 samples) and $\$ 68.00$ (1 sample) for external users. Because speed is an important consideration in disease management, it is not feasible to wait until a 96-well plate is full to run fragment analysis for lineage diagnostics. Therefore, plates generally include far fewer than 96 samples for $P$. infestans lineage determination. Factors such as cost and time are important considerations for choosing a diagnostic technique. The benefit of increased information acquisition, like that achieved through SSR analysis, could be outweighed by the higher costs of time and money. This is especially true when relatively few genotypes are being observed and, therefore, little new information is obtained through SSR analysis.

In cases where acquisition of HRM analysis software or the LNA probes is cost prohibitive, Tm alone may be sufficient for distinguishing genotypes. For example, following amplification by primers Rgn86_1 which target two SNPs, the Tm of US-23 genotypes is consistently $0.9^{\circ} \mathrm{C}$ higher than that of non-US-23 genotypes. Determining sample genotypes using this approach can be done using standard qPCR software. However, this approach may be less feasible for primers targeting SNPs like Rgn88_1, where the Tm difference between genotypes is smaller $\left(0.2^{\circ} \mathrm{C}\right)$.

Since 2012, US-23 has been the predominant clonal lineage reported by USAblight, a national project responsible for studying potato and tomato late blight in the United States (http://www. usablight.org) (Fry et al. 2015). In fact, since 2013, US-23 has accounted for nearly all of the samples analyzed by the USAblight team, and this trend has persisted through the time of this writing (September 2015) (http://www.usablight.org/node/52). A potential drawback to the diagnostic assays described here is that results could be misleading if a new lineage appeared in the United States with the same SNPs as those used in the US-23-specific assays. Although we have tested the lineages that have predominated in the US over recent years, it is possible that a new lineage could appear that shares the US-23 genotype at the diagnostic loci. To address this issue, the assays described here could be used as a preliminary rapid screen for US-23, with an SSR follow-up test to verify that a new lineage is not present. Alternatively, SSR analysis could be used early in the season and, if US-23 was found to be the common lineage, then the HRM or LNA assays could diagnose subsequent samples submitted throughout the season. Presently, late blight samples are shipped from around the country and collected in a central laboratory. There, all samples are analyzed by SSR analysis to determine each sample's lineage. The assays developed in this study could offer more rapid and accessible approaches for determining whether or not a sample belongs to the lineage US-23. This could allow for genotype determination by local diagnostic laboratories, thereby decreasing costs and time between sample submission and diagnostic results.

\section{Acknowledgments}

This work was supported, in part, by the United States Department of Agriculture (USDA) Agricultural Research Service Grant 5358-22000-039-00D (N. J. Grünwald) and USDA National Institute of Food and Agriculture Grant 2011-68004-30154 (H. S. Judelson, C. D. Smart, and N. J. Grünwald). We thank W. Fry (Cornell University) for providing isolates which were sent to his lab for genotyping, and the many researchers and growers across the country who continue to collect and submit isolates.

\section{Literature Cited}

Braasch, D. A., and Corey, D. R. 2001. Locked nucleic acid (LNA): Fine-tuning the recognition of DNA and RNA. Chem. Biol. 8:1-7.

Broad Institute. 2009. Phytophthora infestans Sequencing Project. Online publication. Broad Institute of Harvard and MIT. http://www.broadinstitute.org/ annotation/genome/phytophthora_infestans/MultiHome.html

Bustin, S. A., Benes, V., Garson, J. A., Hellemans, J., Huggett, J., Kubista, M., Mueller, R., Nolan, T., Pfaffl, M. W., Shipley, G. L., Vandesompele, J., and Wittwer, C. T. 2009. The MIQE guidelines: Minimum Information for publication of quantitative real-time PCR experiments. Clin. Chem. 55: 611-622.

Caten, C. E., and Jinks, J. L. 1968. Spontaneous variability of single isolate of Phytophthora infestans. I. Cultural variation. Can. J. Bot. 46:329-348.

Cooke, D. E. L., Cano, L. M., Raffaele, S., Bain, R. A., Cooke, L. R., Etherington, G. J., Deahl, K. L., Farrer, R. A., Gilroy, E. M., Goss, E. M., Grünwald, N. J., Hein, I., MacLean, D., McNicol, J. W., Randall, E., Oliva, R. F., Pel, M. A., Shaw, D. S., Squires, J. N., Taylor, M. C., Vleeshouwers, V.G. A. A., Birch, P. R. J., Lees, A. K., and Kamoun, S. 2012. Genome analyses of an aggressive and invasive lineage of the Irish potato famine pathogen. PLoS Pathog. 8:e1002940.

Danecek, P., Auton, A., Abecasis, G., Albers, C. A., Banks, E., DePristo, M. A., Handsaker, R. E., Lunter, G., Marth, G. T., Sherry, S. T., McVean, G., and Durbin, R. 2011. The variant call format and VCFtools. Bioinformatics 27: 2156-2158.

Danies, G., Myers, K., Mideros, M. F., Restrepo, S., Martin, F. N., Cooke, D. E. L., Smart, C. D., Ristaino, J. B., Seaman, A. J., Gugino, B. K., Grünwald, N. J., and Fry, W. E. 2014. An ephemeral sexual population of Phytophthora infestans in the northeastern United States and Canada. PLoS One 9:e116354.

Danies, G., Small, I. M., Myers, K., Childers, R., and Fry, W. E. 2013. Phenotypic characterization of recent clonal lineages of Phytophthora infestans in the United States. Plant Dis. 97:873-881.

Flier, W. G., Grunwald, N. J., Fry, W. E., and Turkensteen, L. J. 2001. Formation, production and viability of oospores of Phytophthora infestans from potato and Solanum demissum in the Toluca Valley, central Mexico. Mycol. Res. 105: 998-1006.

Fry, W. E., Birch, P. R. J., Judelson, H. S., Grunwald, N. J., Danies, G., Everts, K. L., Gevens, A. J., Gugino, B. K., Johnson, D. A., Johnson, S. B., Mcgrath, M. T., Myers, K. L., Ristaino, J. B., Roberts, P. D., Secor, G., and Smart, C. D 2015. Five reasons to consider Phytophthora infestans a reemerging pathogen. Phytopathology 105:966-981.

Fry, W. E., and Goodwin, S. B. 1997. Re-emergence of potato and tomato late blight in the United States. Plant Dis. 81:1349-1357. 
Fry, W. E., McGrath, M. T., Seaman, A.,Zitter, T. A., McLeod, A., Danies, G., Small, I. M., Myers, K., Everts, K., Gevens, A. J., Gugino, B. K., Johnson, S. B., Judelson, H., Ristaino, J., Roberts, P., Secor, G., Seebold, K., Snover-Clift, K., Wyenandt, A., Grunwald, N. J., and Smart, C. D. 2013. The 2009 late blight pandemic in Eastern USA-Causes and results. Plant Dis. 97:296-306.

Gavino, P. D., Smart, C. D., Sandrock, W., Miller, J. S., Hamm, P. B., Lee, T. Y. Davis, R. M., and Fry, W. E. 2000. Implications of sexual reproduction for Phytophthora infestans in the United States: Generation of an aggressive lineage. Plant Dis. 84:731-735.

Goodwin, S. B., Cohen, B. A., Deahl, K. L., and Fry, W. E. 1994a. Migration from Northern Mexico as the probable cause of recent changes in populations of Phytophthora infestans in the United States and Canada. Phytopathology 84: 553-558.

Goodwin, S. B., Cohen, B. A., and Fry, W. E. 1994b. Panglobal distribution of a single clonal lineage of the Irish potato famine fungus. Proc. Natl. Acad. Sci. USA 91:11591-11595.

Goodwin, S. B., Drenth, A., and Fry, W. E. 1992. Cloning and genetic analyses of two highly polymorphic, moderately repetitive nuclear DNAs from Phytophthora infestans. Curr. Genet. 22:107-115.

Goodwin, S. B., Smart, C. D., Sandrock, R. W., Deahl, K. L., Punja, Z. K., and Fry, W. E. 1998. Genetic change within populations of Phytophthora infestans in the United States and Canada during 1994 to 1996: Role of migration and recombination. Phytopathology 88:939-949.

Goss, E. M., Tabima, J. F., Cooke, D. E. L., Restrepo, S., Fry, W. E., Forbes, G. A., Fieland, V. J., Cardenas, M., and Grünwald, N. J. 2014. The Irish potato famine pathogen Phytophthora infestans originated in central Mexico rather than the Andes. Proc. Natl. Acad. Sci. USA 111:8791-8796.

Haas, B. J., Kamoun, S., Zody, M. C., Jiang, R. H. Y., Handsaker, R. E., Cano, L. M., Grabherr, M., Kodira, C. D., Raffaele, S., Torto-Alalibo, T., Bozkurt, T. O., Ah-Fong, A. M. V., Alvarado, L., Anderson, V. L., Armstrong, M. R., Avrova, A., Baxter, L., Beynon, J., Boevink, P. C., Bollmann, S. R., Bos, J. I. B., Bulone, V., Cai, G., Cakir, C., Carrington, J. C., Chawner, M., Conti, L., Costanzo, S., Ewan, R., Fahlgren, N., Fischbach, M. A., Fugelstad, J., Gilroy, E. M., Gnerre, S., Green, P. J., Grenville-Briggs, L. J., Griffith, J., Grünwald, N. J., Horn, K., Horner, N. R, Hu, C.-H., Huitema, E., Jeong, D.-H., Jones, A. M. E., Jones, J. D. G., Jones, R. W., Karlsson, E. K., Kunjeti, S. G., Lamour, K., Liu, Z., Ma, L., Maclean, D., Chibucos, M. C., McDonald, H., McWalters, J., Meijer, H. J. G., Morgan, W., Morris, P. F., Munro, C. A., O’Neill, K., Ospina-Giraldo, M., Pinzón, A., Pritchard, L., Ramsahoye, B., Ren, Q., Restrepo, S., Roy, S., Sadanandom, A., Savidor, A., Schornack, S., Schwartz, D. C., Schumann, U. D., Schwessinger, B., Seyer, L., Sharpe, T., Silvar, C., Song, J., Studholme, D. J., Sykes, S., Thines, M., van de Vondervoort, P. J. I., Phuntumart, V., Wawra, S., Weide, R., Win, J., Young, C., Zhou, S., Fry, W., Meyers, B. C., van West, P., Ristaino, J., Govers, F., Birch, P. R. J., Whisson, S. C., Judelson, H. S., and Nusbaum, C. 2009. Genome sequence and analysis of the Irish potato famine pathogen Phytophthora infestans. Nature 461:393-398.

Haverkort, A. J., Boonekamp, P. M., Hutten, R., Jacobsen, E., Lotz, L. A. P., Kessel, G. J. T., Visser, R. G. F., and Vossen, E. A. G. 2008. Societal costs of late blight in potato and prospects of durable resistance through cisgenic modification. Potato Res. 51:47-57.

Hu, C.-H., Perez, F. G., Donahoo, R., McLeod, A., Myers, K., Ivors, K., Secor, G., Roberts, P. D., Deahl, K. L., Fry, W. E., and Ristaino, J. B. 2012. Recent genotypes of Phytophthora infestans in the eastern United States reveal clonal populations and reappearance of mefenoxam sensitivity. Plant Dis. 96:1323-1330.

Jaime-Garcia, R., Trinidad-Correa, R., Felix-Gastelum, R., Orum, T. V., Wasmann, C. C., and Nelson, M. R. 2000. Temporal and spatial patterns of genetic structure of Phytophthora infestans from tomato and potato in the Del Fuerte Valley. Phytopathology 90:1188-1195.

Johnson, M. P., Haupt, L. M., and Griffiths, L. R. 2004. Locked nucleic acid (LNA) single nucleotide polymorphism (SNP) genotype analysis and validation using real-time PCR. Nucleic Acids Res. 32:e55.
Kiewnick, S., Frey, J. E., and Braun-Kiewnick, A. 2015. Development and validation of LNA-based quantitative real-time PCR assays for detection and identification of the root-knot nematode Meloidogyne enterolobii in complex DNA backgrounds. Phytopathology 105:1245-1249.

Koshkin, A. A., Singh, S. K., Nielsen, P., Rajwanshi, V. K., Kumar, R., Meldgaard, M., Olsen, E., and Wengel, J. 1998. LNA (locked nucleic acids): Synthesis of the adenine, cytosine, guanine, 5-methylcytosine, thymine and uracil bicyclonucleoside monomers, oligomerisation, and unprecedented nucleic acid recognition. Tetrahedron 54:3607-3630.

Langmead, B., and Salzberg, S. L. 2012. Fast gapped-read alignment with Bowtie 2. Nat. Methods 9:357-359.

Lees, A. K., Wattier, R., Shaw, D. S., Sullivan, L., Williams, N. A., and Cooke, D. E. L. 2006. Novel microsatellite markers for the analysis of Phytophthora infestans populations. Plant Pathol. 55:311-319.

Legard, D. E., Lee, T. Y., and Fry, W. E. 1995. Pathogenic specialization in Phytophthora infestans: Aggressiveness on tomato. Phytopathology 85 1356-1361.

Lehner, M. S., Paula Junior, T. J., Silva, R. A., Vieira, R. F., Carneiro, J. E. S., Schnabel, G., and Mizubuti, E. S. G. 2015. Fungicide sensitivity of Sclerotinia sclerotiorum: A thorough assessment using discriminatory dose, EC 50, high-resolution melting analysis, and description of new poin mutation associated with thiophanate-methyl resistance. Plant Dis. 99: 1537-1543.

Li, H., Handsaker, B., Wysoker, A., Fennell, T., Ruan, J., Homer, N., Marth, G., Abecasis, G., and Durbin, R. 2009. The sequence alignment/map format and SAMtools. Bioinformatics 25:2078-2079.

Martin, M. D., Cappellini, E., Samaniego, J. A., Zepeda, M. L., Campos, P. F., Seguin-Orlando, A., Wales, N., Orlando, L., Ho, S. Y. W., Dietrich, F. S., Mieczkowski, P. A., Heitman, J., Willerslev, E., Krogh, A., Ristaino, J. B., and Gilbert, M. T. P. 2013. Reconstructing genome evolution in historic samples of the Irish potato famine pathogen. Nat. Commun. 4:2172.

McKenna, A., Hanna, M., Banks, E., Sivachenko, A., Cibulskis, K., Kernytsky, A., Garimella, K., Altshuler, D., Gabriel, S., Daly, M., and DePristo, M. 2010. The Genome Analysis Toolkit: A MapReduce framework for analyzing nextgeneration DNA sequencing data. Genome Res. 20:1297-1303.

National Center for Biotechnology Information. 2012. Primer-BLAST-A tool for finding specific primers. Online publication. http://www.ncbi.nlm.nih.gov/ tools/primer-blast/

Raffaele, S., Farrer, R. A., Cano, L. M., Studholme, D. J., MacLean, D. Thines, M., Jiang, R. H. Y., Zody, M. C., Kunjeti, S. G., Donofrio, N. M., Meyers, B. C., Nusbaum, C., and Kamoun, S. 2010. Genome evolution following host jumps in the Irish potato famine pathogen lineage. Science 330:1540-1543.

Reed, G. H., Kent, J. O., and Wittwer, C. T. 2007. High-resolution DNA melting analysis for simple and efficient molecular diagnostics. Pharmacogenomics 8 : 597-608

Stajich, J. E., Harris, T., Brunk, B. P., Brestelli, J., Fischer, S., Harb, O. S., Kissinger, J. C., Li, W., Nayak, V., Pinney, D. F., Stoeckert, C. J. J., and Roos, D. S. 2012. FungiDB: An integrated functional genomics database for fungi. Nucleic Acids Res. 40:D675-D681.

Summers, C. F., Adair, N. L., Gent, D. H., McGrath, M. T., and Smart, C. D. 2015 Pseudoperonospora cubensis and $P$. humuli detection using species-specific probes and high definition melt curve analysis. Can. J. Plant Pathol. 37:315-330.

Tamura, K., Stecher, G., Peterson, D., Filipski, A., and Kumar, S. 2013. MEGA6: Molecular evolutionary genetics analysis version 6.0. Mol. Biol. Evol. 30: $2725-2729$.

Turner, S. R. 2005. After the famine: Plant pathology, Phytophthora infestans, and the late blight of potatoes, 1845-1960. Hist. Stud. Phys. Biol. Sci. 35:341-370.

Yoshida, K., Schuenemann, V. J., Cano, L. M., Pais, M., Mishra, B., Sharma, R. Lanz, C., Martin, F. N., Kamoun, S., Krause, J., Thines, M., Weigel, D., and Burbano, H. A. 2013. The rise and fall of the Phytophthora infestans lineage that triggered the Irish potato famine. eLife 2:e00731. 